Título artículo / Títol article: Experimental Demonstration of the Quasi-Direct Space-to-Time Pulse Shaping Principle

Autores / Autors

Vincent Loriot, Omel Mendoza-Yero, Gladys Mínguez-Vega, Luis Bañares, Rebeca de Nalda

Revista:

IEEE PHOTONICS TECHNOLOGY LETTERS

(2012), VOL. 24, NO. 4

Versión / Versió:

Cita bibliográfica / Cita bibliogràfica (ISO 690):
Postprint del autor

LORIOT, Vicent; MENDOZA-YERO, Omel; MÍNGUEZ-VEGA, Gladys; BAÑARES, Luis; NALDA, Rebeca de. Experimental Demonstration of the Quasi-Direct Space-to-Time Pulse Shaping Principle. IEEE Photonics Technology Letters, 2012, vol. 24, no. 4, p. 273-275. $\underline{\text { http://hdl.handle.net/10234/72746 }}$ 


\title{
Experimental Demonstration of the Quasi-Direct Space-to-Time Pulse Shaping Principle
}

\author{
Vincent Loriot, Omel Mendoza-Yero, Gladys Mínguez-Vega, Luis Bañares and Rebeca de Nalda
}

\begin{abstract}
A new strategy of pulse shaping based on the quasi-direct space-to-time (QDST) conversion principle has been recently proposed theoretically [Mínguez-Vega et al., Opt. Exp. $16,16993(2008)]$. In the present letter, the working principle of this pulse shaper has been experimentally verified by using a simple second order cross-correlation setup with spatial resolution. The QDST conversion principle is evidenced through the measurement of the delay between the different components of the spatial profile. The temporal profile at the focus is shown to follow the spatial profile using the QDST conversion law.
\end{abstract}

Index Terms-Diffractive optics, optical pulse shaping.

$\mathbf{F}$ EMTOSECOND pulse shaping techniques have been used for a variety of applications involving either the control of physical systems with light (see [1] and references therein) or the manipulation of waveforms for optical communications [2]. Different pulse shaping and ultrashort laser pulse characterization techniques have been developed over the years (see [3], [4] and references therein). Recently, new pulse shapers, based on the space-to-time conversion principle, have been proposed. In those, the tailoring of the temporal pulse is controlled through the spatial modulation of the pulse profile. The direct space-to-time (DST) pulse shaper [2], [5] is characterized by a linear relationship between space and time, while for the quasi-direct space-to-time (QDST) pulse shaper [6], [7] , the relationship is quadratic. A QDST pulse shaper consists of a mask attached to a kinoform diffractive lens (DL). The operation principle of the QDST pulse shaper is to focus the radial component of the beam profile at different time delays using the DL and control the temporal shape at the focus by modulating the spatial profile at the entrance of the DL. Characterization of shaped pulses from a QDST pulse shaper carries intrinsically the difficulty of the nonuniformity of the temporal profile along the spatial profile of the pulse in the far field region. Up to now, only spectral measurements at the focus have been experimentally carried out [8]. In this letter, we show experimentally the spatio-

Manuscript received XXX 00, 2011; revised XXX 00, 2011; accepted XXX 00, 2011. Date of publication XXX 00, 000 date of current version XXX 00, 000 .

V. Loriot and R. de Nalda are with the Instituto de Química Física Rocasolano, CSIC, C/Serrano 119, 28006 Madrid, Spain (e-mail: loriot@iqfr.csic.es).

O. Mendoza-Yero, G. Mínguez-Vega are with Institut de Noves Tecnologies de la Imatge (INIT), Universitat Jaume I, 12080 Castelló, Spain and GROC.UJI, Departament de Física, Universitat Jaume I, 12080 Castelló, Spain.

L. Bañares is with Departamento de Química Física I, Facultad de Ciencias Químicas, Universidad Complutense de Madrid, 28040 Madrid, Spain.

Color versions of one or more of the figures in this letter are available online at http://ieeexplore.ieee.org.

Digital Object Identifier 00.0000/LPT.2011.0000000 temporal conversion of the QDST pulse shaper. A simple second-order cross-correlation of the shaped pulse is employed to show the relative delay between the spatial components of the pulse. The technique allows the reconstruction of the QDST shaped pulses at a fix plane from the DL with a simple delay scan and retrieves its temporal shape at the focus.

The scheme of our experimental setup (Fig. 1) has mainly four parts, the QDST pulse shaper branch, the reference beam, the nonlinear gate and the detection system. By using the nonlinear gate, based on a crosscorrelation technique, between the shaped pulse from the QDST and a much shorter broadarea reference pulse we were able to obtain a 3D intensity map of the shaped pulses after the nonlinear crystal. With this information it is easy to retrieve the temporal shape at the focus. The femtosecond laser source used for this purpose is a commercial Ti:sapphire chirped-pulse amplified system delivering $\tau \approx 70$ fs laser pulses centered around $800 \mathrm{~nm}$ and maximum of $3.5 \mathrm{~mJ}$ per pulse at a repetition rate of $1 \mathrm{KHz}$. The incoming femtosecond pulse is conveniently attenuated using a glass wedge and split into two arms using a glass plate. A telescope composed of two lenses $\left(f_{a}=-75 \mathrm{~mm}\right.$ and $\mathrm{f}_{\mathrm{b}}=300 \mathrm{~mm}$ ) is placed on the strongest beam (typically, few tens of $\mu J$ ) to illuminate the DL [9] with the most homogeneous spatial profile as possible. The DL introduces a temporal delay at the focus between the different spatial components of the beam [6], which means that the pulse duration significantly increases. The spatial intensity profile is then modulated using a lithographic mask to change the intensity of the spatial components and thus modulate the temporal profile. The reference beam, with a lower energy (typically, few $\mu J$ ), passes through a motorized optical delay line to control its relative delay with respect to the main beam and focussed using a $f_{p r}=200 \mathrm{~mm}$ lens. The two beams overlap at their focus with a small angle $\left(\approx 13^{\circ}\right)$ on a $\beta-\mathrm{BaB}_{2} \mathrm{O}_{4}(\mathrm{BBO})$ AR coated at $800 \mathrm{~nm}$ and $400 \mathrm{~nm}$ type-I crystal $\left(8 \times 10 \times 0.2 \mathrm{~mm}^{3}, \theta=29.18^{\circ}\right)$, where second harmonic $(\mathrm{SH})$ generation is produced. We have numerically checked that the efficiency of wavelength conversion does not vary by more than $15 \%$ due to the phase matching for the range of incidence angles sampled. To improve the angular resolution of the phase matching in the BBO crystal between the shaped pulse and the reference pulse, an iris is placed on the reference beam to reduce the numerical aperture and increase the confocal parameter. The phase-matched SH signal is recorded by a CCD camera as a function of the time delay between the pulses. Contrary to the theoretical work of [6], no pinhole at the focus of the DL is required for pump-probe type 
experiments because the interaction is limited by the spatial profile of the reference beam. In the present case, in order to be sensitive to spatial effects, the focal size of the reference beam is chosen to be larger than the central mode of the QDST pulse at the focus.

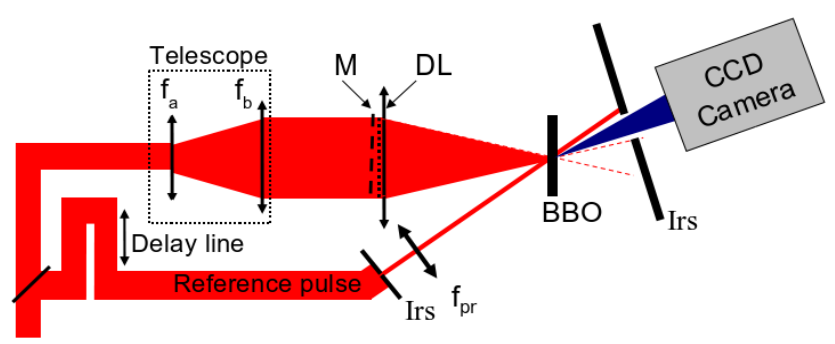

Fig. 1. Experimental setup for the spatially-resolved second order crosscorrelation of a QDST shaped pulse. $f_{a}$ and $f_{b}$ are lenses for the telescope employed to obtain the most uniform spatial profile at the entrance of the QDST pulse shaper. M is the mask, DL is the kinoform diffractive lens, Irs is the iris and $\mathrm{f}_{\mathrm{pr}}$ is the lens placed in the reference beam. BBO is the nonlinear crystal used for $\mathrm{SH}$ generation between phase-matched shaped and reference pulses.

Typical SH images recorded by the CCD camera without inserting any mask before the DL, at two different time delays (440 fs and $1.2 \mathrm{ps}$, respectively) within the temporal overlap between the shaped and reference pulses, are shown in Figs. 2(a) and 2(c). The corresponding angular integrations of the images are depicted in Figs. $2(b)$ and $2(d)$. The images
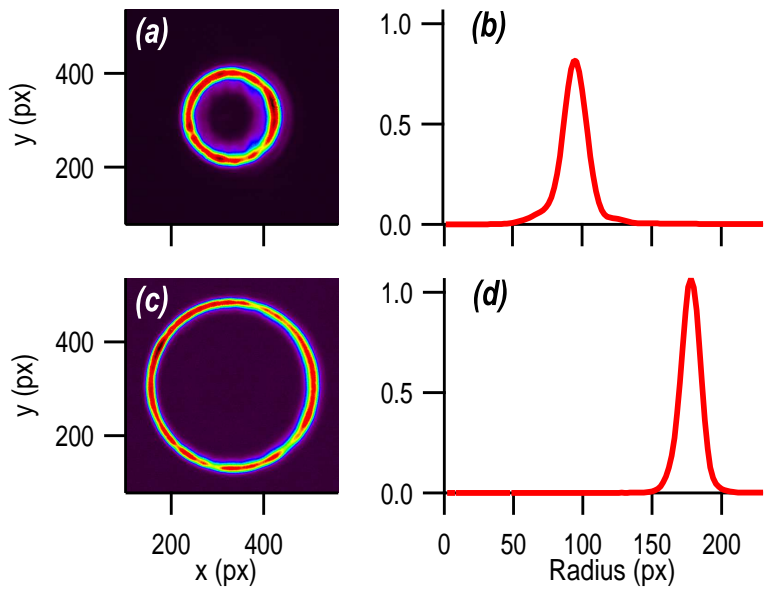

Fig. 2. SH images, in pixel (px) units, recorded on the CCD camera when no mask is inserted before the DL at (a) 440 fs and (c) 1.2 ps time delay between the shaped and reference pulses. Angular integration of the images are shown in $(b)$ and $(d)$, respectively.

show an isotropic ring whose diameter increases with time delay while its width decreases. According to the QDST conversion principle [6], the radius of the ring follows the law

$$
t=\alpha r^{2}
$$

where $t$ is the time delay and $\alpha$ the space-to-time conversion factor. At a fixed time delay between the main and reference pulses, the SH signal at a fix distance after the BBO can be interpreted as the cross-correlation between a spatial ring profile pulse and the reference pulse both assumed to have a temporal
Gaussian shape. Hence, the phase matching produces a signal with ring geometry. The isotropy of the intensity distribution across the ring is a proof that the angular range sampled is within the phase matching region. Upon the derivation of (1), the width of the ring evolves as $\Delta r=t /(2 \alpha r)$. The width of the ring decreases as its radius increases, as observed in Fig. 2. The evolution of the radial distribution as a function of time delay without mask is depicted in Fig. 3(a).
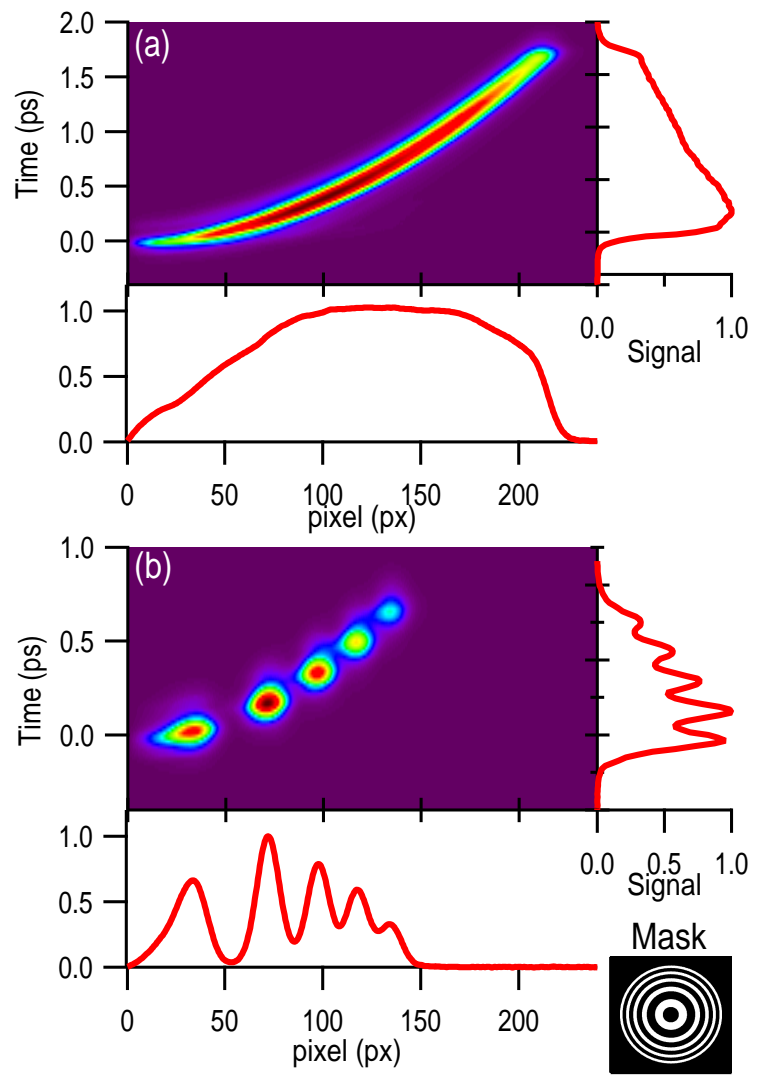

Fig. 3. The 2D plots show the temporal evolution of the radial distribution obtained by angular integration of the $\mathrm{SH}$ images as a function of the time delay between shaped and reference pulses. (a) Without a mask before the DL. (b) Using the mask shown at the right bottom part of the figure. Radial (in pixels) and temporal integration graphs of the images are shown to the right and below in each case.

We have carried out a two-dimensional fit of the images shown in Fig. 3 using the following equation

$$
\begin{aligned}
f(r, t) \propto & \left\{I_{r}(r) \otimes\left[r I_{\mathrm{sp}}(r) H\left(r_{\max }-r\right)\right]\right\} \\
& \times \exp \left(-4 \ln 2 \frac{\left(t-\alpha r^{2}\right)^{2}}{\tau_{a}^{2}}\right),
\end{aligned}
$$

where $I_{r}(r)$ is the spatial optical resolution of the system (assumed to have a Gaussian shape) modeling the $\vec{k}$ vector dispersion of the reference pulse, $I_{\mathrm{sp}}(r)$ the radial profile of the pulse before the DL, $H(r)$ the Heaviside function which delimits the maximum $\mathrm{SH}$ ring radius $r_{\max }$ measured on the CCD camera and $\tau_{a}$ is the autocorrelation time assumed to be constant over the radial profile before the DL. From this last term, it is possible to estimate the pulse duration to be $\tau=89 \pm 3 \mathrm{fs}$, which is consistent with the laser duration 
including the potential error over the optical resolution $I_{r}(r)$. Since the fit determines $\alpha=(37.65 \pm 0.30) \times 10^{-3} \mathrm{fs} / \mathrm{px}^{2}$ and $r_{\max }=216 \pm 1 \mathrm{px}$, the incoming temporal profile is spread over a range of $\Delta t_{\text {str }}=\alpha r_{\max }^{2}=1.75 \pm 0.03 \mathrm{ps}$. This value is found to be in very good agreement with the value obtained from the radius of the lens $\left(r_{\mathrm{f}}=10.60 \pm 0.05 \mathrm{~mm}\right)$ and the focal length [9] $\left(Z_{0}=106 \mathrm{~mm}\right.$ at $\left.800 \mathrm{~nm}\right)$, which gives $\Delta t_{\text {str }}=r_{\mathrm{f}}^{2} /\left(2 c Z_{0}\right)=1.77 \pm 0.02$ ps.

The ability of the QDST pulse shaper to generate custom shaped pulses is shown in Fig. 3 as characterized by the present spatio-temporal methodology. A mask with concentric rings with a radial binary transmittance $\Gamma(r)$ (shown as an inset in Fig. 3(b)) was placed before the DL. For the experiment, we manufactured the mask by photolithography with direct laser writing on a chrome photomask. Our laser writing machine (Microtech, Palermo, Italy) allows us to obtain diffractive masks with a global resolution of $0.2 \mu \mathrm{m}$ and a minimum feature size of $0.8 \mu \mathrm{m}$. The expected electric field at the focus $E_{\text {foc }}(t)$ can be written as

$E_{\text {foc }}(t)=\exp \left(-4 \ln 2(t / \tau)^{2}\right) \otimes\left(\Gamma\left(t=\alpha r^{2}\right) E_{\mathrm{sp}}\left(t=\alpha r^{2}\right)\right)$

where $\Gamma(t)$ and $E_{\mathrm{sp}}(t)$ are, respectively, the transmittance of the mask and the spatial profile expressed in the temporal domain using eq. (1). The cross-correlation $S(t)$, i.e. the radial and angular integration of the images, reflects the modulation of the mask and produce a signal given by $S(t) \propto\left(E_{\mathrm{ref}}(t) \otimes E_{\mathrm{foc}}(t)\right)^{2}$, where $E_{\mathrm{ref}}(t)$ is the temporal profile of the reference pulse. The signal $S(t)$ is illustrated with and without mask in the side plots of Fig. 3.

The QDST shaped pulse can be visualized using the present characterization method, both in space and time, by representing the iso-intensity of the spatio-temporal resolved data as shown in Fig. 4. At fix plane after the DL, each point of the spatial profile of the QDST transformation can be seen as the $2^{\text {nd }}$ order autocorrelation of the laser pulse. The 3D visualization makes evident the QDST conversion principle written in (1). It appears very clear that the central part arrives before the surrounding part and the relative delay of the spatial profile has a quadratic dependence with respect to time. The modulation of the spatial profile thus induces a shaped pulse at the focus. Since the entire signal has been generated at the focus, the temporal profile of the pulse at the focus is approximatively the integration of the signal collected on the camera.

In summary, using a simple spatially resolved second order cross-correlation method, it is possible to observe the space-totime relation induced by the DL and the possibility to generate a custom made shaped pulse, in a very well defined temporal window, using a concentric ring geometry mask. A 2D-SLM mask could be easily implemented in the QDST pulse shaper in order to produce arbitrary shaped pulses applicable in adaptive quantum control experiments. Work is in progress in our groups in that direction.

\section{ACKNOWLEDGMENT}

V.L. thanks the Consejo Superior de Investigaciones Cientificas (CSIC) for ER and JAE-DOC postdoctoral contracts,
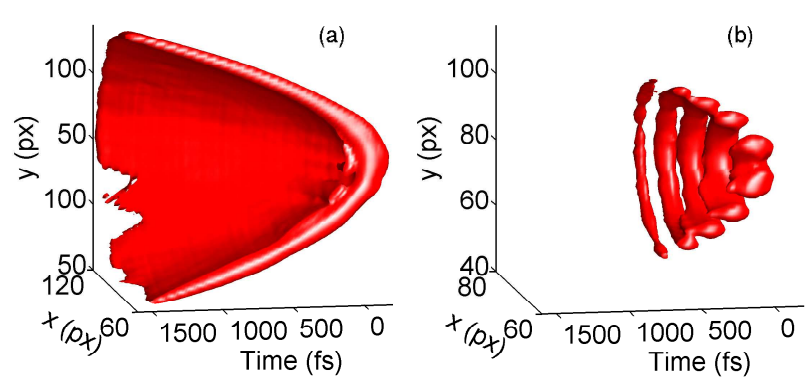

Fig. 4. 3D representation of the iso-intensity surface at the intensity level of $\left(I_{\max } / 20\right)$ obtained by cross-correlation when $(a)$ no mask is present before the DL and $(b)$ when a concentric-ring structure mask is located before the DL (shown in Fig.3). A cut of the 3D structure along the $(y$-time) plane is shown to better visualize the internal geometrical aspect of the shaped pulses.

respectively. This work has been supported by the Spanish Ministry of Science and Innovation (MCINN) through grant CTQ2008-02578/BQU, the Consolider program "Science and Applications of Ultrafast Ultraintense Lasers" grant no. CSD2007-00013 and the European ITN Program FASTQUAST grant PITN-GA-2008-214962. This research has been carried out within the Unidad Asociada "Química Física Molecular" between Departamento de Química Física I of Universidad Complutense de Madrid (UCM) and CSIC. The facilities provided by the Centro de Asistencia a la Investigación de Espectroscopia Multifotónica y de Femtosegundo (UCM) are gratefully acknowledged. The authors are very grateful to E. Tajahuerce and the Serveis Centrals d'Intrumentació Científica (SCIC) of the Universitat Jaume I for the mask fabrication. J. R. Vázquez de Aldana is gratefully acknowledged for fruitful discussions.

\section{REFERENCES}

[1] P. Nuernberger, G. Vogt, T. Brixner, and G. Gerber, "Femtosecond quantum control of molecular dynamics in the condensed phase," Phys. Chem. Chem. Phys., vol. 9, pp. 2470-2497, 2007.

[2] D. E. Leaird and A. M. Weiner, "Femtosecond optical packet generation by a direct space-to-time pulse shaper," Opt. Lett., vol. 24, no. 12, pp. 853-855, 1999.

[3] A. Monmayrant, S. Weber, and B. Chatel, "A newcomer's guide to ultrashort pulse shaping and characterization," Journal of Physics B: Atomic, Molecular and Optical Physics, vol. 43, no. 10, p. 103001, 2010.

[4] A. M. and Weiner, "Ultrafast optical pulse shaping: A tutorial review," Optics Communications, vol. 284, no. 15, pp. 3669 - 3692, 2011.

[5] T. Mansuryan, M. Kalashyan, J. Lhermite, E. Suran, V. Kermene, A. Barthelemy, and F. Louradour, "Compact direct space-to-time pulse shaping with a phase-only spatial light modulator," Opt. Lett., vol. 36, no. 9, pp. 1635-1637, 2011.

[6] G. Mínguez-Vega, O. Mendoza-Yero, J. Lancis, R. Gisbert, and P. Andrés, "Diffractive optics for quasi-direct space-to-time pulse shaping," Opt. Express, vol. 16, no. 21, pp. 16993-16998, 2008.

[7] O. Mendoza-Yero, G. Mínguez-Vega, J. Lancis, and V. Climent, "Diffractive pulse shaper for arbitrary waveform generation," Opt. Lett., vol. 35 , no. 4, pp. 535-537, 2010.

[8] G. Minguez-Vega, O. Mendoza-Yero, E. Tajahuerce, J. Lancis, and P. Andres, "Optical filter based on a spatially patterned kinoform diffractive lens," Photonics Technology Letters, IEEE, vol. 21, no. 6, pp. 347 -349, march15, 2009.

[9] G. Mínguez-Vega, C. Romero, O. Mendoza-Yero, J. R. V. de Aldana, R. Borrego-Varillas, C. Méndez, P. Andrés, J. Lancis, V. Climent, and L. Roso, "Wavelength tuning of femtosecond pulses generated in nonlinear crystals by using diffractive lenses," Opt. Lett., vol. 35, no. 21, pp. 3694-3696, 2010. 